\title{
Verifying Electronic Control Units - today and tomorrow
}

\section{(Invited Lecture)}

\author{
W. Damm \\ OFFIS, Embedded Systems Division, Germany \\ werner@egk.informatik.uni-oldenburg.de
}

\begin{abstract}
Electronic Control Units (ECUs) are in almost every non-trivial physical system you use today - automotive, telecommunication, avionics, you name it. Software design for ECUs that gives life to such systems is growing exponentially, with ECU development costs even dominating development costs for the total system in some domains. For example, today's cars contain $60-100$ ECUs, whose development costs are $12-35 \%$ of the total cost.

ECU designs constitute an excellent challenge for formal methods. They often realize safety critical functions, need to deliver their response under hard real-time constraints, are most often hybrid in nature, and are increasingly realized in a distributed fashion.

This talk reports on results on verifying industrial ECU designs both in avionics and automotive and discusses challenges in extending the power of the currently available verification technology.
\end{abstract}

\section{BIOGRAPHY}

Dr. Werner Damm received his Ph.D. in computer science at the RWTH Aachen, Germany, in 1981. He has been a professor in the Computer Science Department, the University of Oldenburg, since 1987 and holds the Chair for Computer Architecture. He is member of the Board of Directors of the OFFIS research institute, which is dedicated to technology transfer, in particular in the domain of embedded systems. He has been leading several industrial projects within OFFIS on hardware verification and verification of safety critical embedded systems. His research interests include multiprocessor systems, semiformal specification methods, synthesis, and verification. He has been serving on program committees of the CAV, CHARME, CHDL, PARLE and EURO-VHDL conferences, is an associate editor of the Journal on Formal Methods in System Design, and is a member of IFIP WG10.5. 